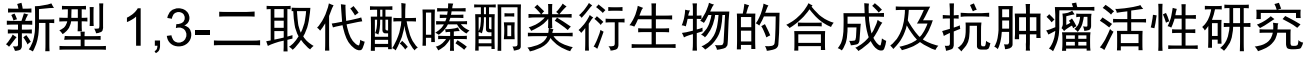

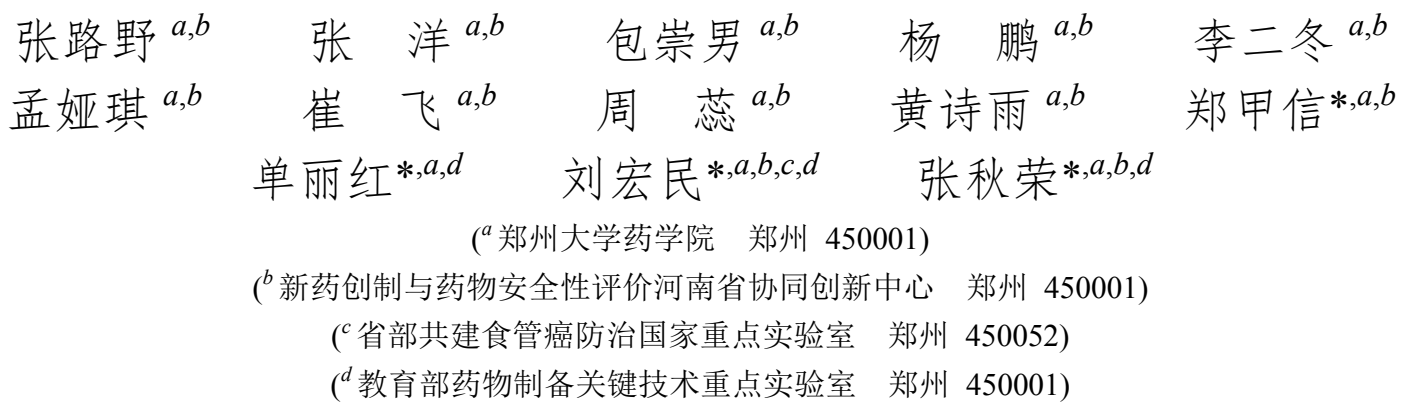

\begin{abstract}
摘要 为了寻找高效低毒的抗肿瘤药物, 设计并合成新型的 1,3 位取代酞嗪酮类化合物. 采用噻唑蓝(MTT)法对目标化 合物在 MCF-7(人乳腺癌细胞)、PC-3(人前列腺癌细胞)、SW-620(人结肠癌细胞)和 HGC-27(人胃癌细胞)四种人类癌细 胞的抗增殖活性进行评价. 结果显示大部分化合物具有较好的抗增殖活性. 其中, 2-(4-(4-澳苯基)-1-氧代唒嗪-2 $(1 H)$ 基)- $N$-(2-氟苯基)乙酰胺 $(\mathbf{5 g})$ 对 MCF-7 细胞的抗增殖活性较好, $\mathrm{IC}_{50}$ 值为 $6.01 \mu \mathrm{mol} / \mathrm{L}$, 为抗肿瘤药物的研究提供了思路. 关键词 酞嗪; 衍生物; 合成; 抗增殖活性
\end{abstract}

\section{Synthesis and Antitumor Activity of Novel 1,3-Disubstituted Pyridazinone Derivatives}

\author{
Zhang, Luye $\mathrm{e}^{a, b}$ \\ Zhang, Yang ${ }^{a, b}$ \\ Bao, Chongnan ${ }^{a, b}$ \\ Yang, Peng ${ }^{a, b}$ \\ Li, Erdong ${ }^{a, b}$ \\ Meng, Yaqi ${ }^{a, b}$ \\ Cui, Fei ${ }^{a, b}$ \\ Zhou, Rui ${ }^{a, b}$ \\ Huang, Shiyu ${ }^{a, b}$ \\ Zheng, Jiaxin $*, a, b$ \\ Shan, Lihong ${ }^{*, a, d} \quad$ Liu, Hongmin ${ }^{*, a, b, c, d}$ Zhang, Qiurong ${ }^{*, a, b, d}$ \\ ( ${ }^{a}$ School of Pharmaceutical Sciences, Zhengzhou University, Zhengzhou 450001) \\ $\left({ }^{b}\right.$ Collaborative Innovation Center of New Drug Research and Safety Evaluation, Zhengzhou 450001) \\ ( ${ }^{c}$ State Key Laboratory of Esophageal Cancer Prevention \& Treatment, Zhengzhou University y, Zhengzhou 450052) \\ ( ${ }^{d}$ Key Laboratory of Advanced Drug Preparation Technologies, Ministry of Education of China, Zhengzhou 450001)
}

\begin{abstract}
In order to find more efficient and low toxicity antitumor drugs, a series of novel 1,3-disubstituted pyridazinone derivatives were synthesized and evaluated for their antiproliferative activities against four human cancer cell lines (MCF-7, PC-3, SW-620 and HGC-27) in vitro. The results showed that most compounds had good antiproliferative activities, especially 2-(4-(4-bromophenyl)-1-oxo-tolylazine-2(1H)-yl)- $N$-(2-fluorophenyl)acetamide (5g) exhibited better antiproliferative activities with $\mathrm{IC}_{50}$ value of $6.01 \mu \mathrm{mol} / \mathrm{L}$. In a nutshell, this work provided clues to discover antitumor agent based on the quinazoline scaffold.
\end{abstract}

Keywords pyridazinederiv; ative; synthesis; antiproliferative activity

肿瘤尤其是恶性肿瘤，是导致人类疾病死亡的 “第 二大杀手”，据预测, 2035 年全球癌症死亡人数将增加 到 1315 万人 ${ }^{[1,2]}$. 恶性肿瘤的致死因素主要表现为两个
方面: 一是癌细胞不受控制的疯狂生长, 二是癌细胞逐 渐侵入周围正常组织，入侵并破坏邻近组织，有时会转 移，即通过淋巴或血液扩散到身体其它位置 ${ }^{[3]}$. 现有的

\footnotetext{
* Corresponding authors. E-mail: zqr409@yeah.net; shlh@zzu.edu.cn; liuhm@zzu.edu.cn; zjx1224@163.com Received August 8, 2019; revised October 11, 2019; published online November 21, 2019.

Project supported by the National Natural Science Foundation of China (No. 81430085), the Natural Science Foundation of Henan Province (No. 182300410321), and the Openning Fund from State Key Laboratory of Esophageal Cancer Prevention \& Treatment (No. K2020000X).

国家自然科学基金(No. 81430085)、河南省自然科学基金(No.182300410321)、省部共建食管癌防治国家重点实验室开放基金(No. K2020000X)资助项 目.
} 
肿瘤治疗方案包括手术治疗、化学治疗和放射治疗等 ${ }^{[4]}$. 在癌症的治疗过程中, 化学药物治疗在临床上是一种非 常重要的方法. 目前常用的抗肿瘤药物具有不同程度的 选择性差、毒副作用、不良反应多和易产生耐药性等缺 点 ${ }^{[5]}$, 因此, 设计并合成高效安全的抗肿瘤药物对肿瘤 的治疗有重要意义 ${ }^{[6,7]}$.

酞嗪杂环是一种䒺环的 2,3 位碳被氮原子取代而形 成的化合物 ${ }^{[8]}$. 在大量含氮杂环的有机化合物中, 酞嗪

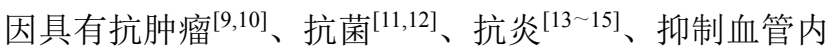
皮生长因子受体 II (VEGFR-2)等药理作用和临床价值 而受到很多广大科研工作者的关注 ${ }^{[16,17]}$, 目前酞嗪类化 合物有的已经上市或正处于临床期研究. 奥拉帕尼 (Olaparib, 图 1) 由英国阿斯利康公司研发, 是一种选择 性的 PARP1/2 抑制剂, 经美国食品药品监督管理局 (FDA)批准用于治疗卵巢癌, 但在临床应用过程中部分 患者出现贫血、恶心和乏力等不良反应 ${ }^{[18]}$. 琥珀酸瓦他 拉尼(Vatalanib succinate, 图 1)由 Novartis 和 Schering AG 公司合作开发的选择性 VEGFR-2 抑制剂 ${ }^{[19]}$, 主要用于 转移性结直肠癌和前列腺癌的治疗, 目前正处于临床三 期研究阶段.
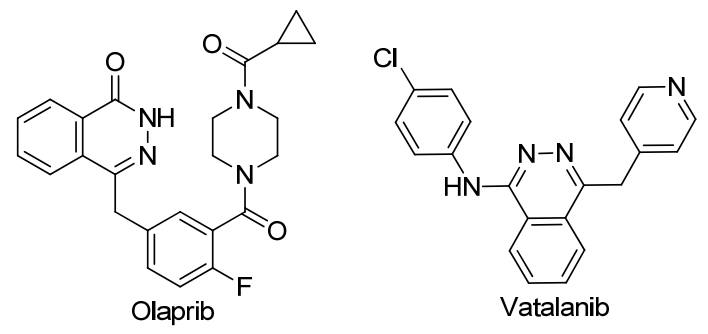

图 1 含唒嗪环基团药物的分子结构

Figure 1 Structures of drug containing pyridazine ring group

酰胺键是羧酸分子上的羟基被氨基或着胺苯基取 代后所形成的一种化学键 ${ }^{[20]}$. 酰胺结构在化合物结构 的连接过程中起着极为重要的作用 ${ }^{[21]}$, 它是蛋白质的 基本构成结构之一, 对生命科学的发展起着重要作用.
许多的临床药物分子结构中含有酰胺键, 它具有延长药 物的作用时间，提高药物的生物利用度以及降低药物的 毒副作用等作用 ${ }^{[22]}$.

根据上述酞嗪和酰胺的结构特征，基于本课题组的 研究基础和文献调研，以奥拉帕尼为先导化合物，将酰 胺结构引入酞嗪母核中，设计并合成了一系列新型的 1,3-二取代酞嗪酮类衍生物, 并对其抗肿瘤活性进行测 试和评价.

\section{1 结果与讨论}

\section{1 目标化合物的合成}

新型 1,3-二取代的酞嗪酮类衍生物的合成路线见 Scheme 1. 以邻苯二甲酸酐和溴苯为原料, 氯化铝作催 化剂，制备酞嗪母核(化合物 2). 化合物 $\mathbf{2}$ 和水合肼在无 水乙醇中，以浓硫酸作催化剂制备得到中间体 3. 带不 同取代基的苯胺和氯乙酰氯在二氯甲烷中反应，制备得 到中间体 $4 a \sim 4 r$. 然后中间体 $4 a \sim 4 r$ 和 3 在二氯甲烷 中反应得到目标化合物 $\mathbf{5 a} \sim \mathbf{5 r}$. 所有化合物均通过 ${ }^{1} \mathrm{H}$ $\mathrm{NMR},{ }^{13} \mathrm{C} \mathrm{NMR}$ 和 H RMS 技术进行了结构确证.

\section{2 目标化合物的抗增殖活性}

对表 1 进行分析，该系列化合物对四种癌细胞中的 MCF-7 细胞的活性良好, 而对于其余三种肿瘤细胞的 活性较差. 对该系列化合物的构效关系进行研究, 当该 系列化合物苯环上无取代基时(如化合物 $\mathbf{5 h}$ ), 基本无抗 增殖活性; 当苯环替换为体积更大的菜环时，化合物 $5 \mathrm{~m}$ 的活性良好, 其 $\mathrm{IC}_{50}$ 达到了 $7.89 \mu \mathrm{mol} / \mathrm{L}$, 优于对照 药物氟尿嘧啶的活性 $\left(\mathrm{IC}_{50}=14.78 \mu \mathrm{mol} / \mathrm{L}\right)$; 当取代基 $\mathrm{R}$ 是烷基时, 如化合物 $\mathbf{5 a}$ 和 $\mathbf{5 e}$ 的 $\mathrm{IC}_{50}$ 值分别为 7.72 和 $14.69 \mu \mathrm{mol} / \mathrm{L}$, 说明苯环上的氢被烷基取代后，该系列 化合物的活性增强; 当苯环上的氢被硝基或者烷氧基取 代时，如化合物 $5 b 、 5 d 、 5 f$ 和 $5 k$ 等，活性较差，说明苯 环上有硝基或烷氧基时，对化合物的活性没有任何帮 助; 当取代基 $\mathrm{R}$ 为单取代的卤素时，如化合物 5c、5<smiles>[R]c1ccccc1NC(=O)Cn1nc(-c2ccc(Br)cc2)c2ccccc2c1=O</smiles>

图式 1 化合物 5a $\sim 5 r$ 的合成路线

Scheme 1 Synthesis of compounds $5 \mathbf{a} \sim 5 \mathbf{r}$ 
表 1 化合物 $\mathbf{5 a} \sim 5 \mathbf{r}$ 对四种人类癌细胞的体外抗增殖活性

Table 1 Antiproliferative activity of target compounds $5 \mathbf{a} \sim 5 \mathbf{r}$ against four cancer cell lines

\begin{tabular}{|c|c|c|c|c|c|}
\hline \multirow{2}{*}{ 化合物 } & \multirow{2}{*}{$\mathrm{R}$} & \multicolumn{4}{|c|}{$\mathrm{IC}_{50}{ }^{a} /\left(\mu \mathrm{mol} \cdot \mathrm{L}^{-1}\right)$} \\
\hline & & MCF-7 & PC-3 & SW-620 & HGC-27 \\
\hline $5 a$ & $2-\mathrm{CH}_{2} \mathrm{CH}_{3}$ & $7.18 \pm 0.87$ & $36.91 \pm 0.92$ & $>50$ & $41.52 \pm 1.02$ \\
\hline $5 \mathbf{b}$ & $2-\mathrm{NO}_{2}$ & $22.76 \pm 1.36$ & $24.12 \pm 0.77$ & $>50$ & $26.21 \pm 1.15$ \\
\hline $5 c$ & $3-\mathrm{F}$ & $11.10 \pm 0.98$ & $27.41 \pm 0.23$ & $37.58 \pm 1.47$ & $32.31 \pm 1.01$ \\
\hline $5 d$ & $4-\mathrm{NO}_{2}$ & $>50$ & $39.61 \pm 0.81$ & $>50$ & $34.95 \pm 1.32$ \\
\hline $5 e$ & $3-\mathrm{CH}_{3}$ & $14.68 \pm 1.17$ & $>50$ & $>50$ & $>50$ \\
\hline $5 f$ & $3-\mathrm{NO}_{2}$ & $>50$ & $31.46 \pm 0.80$ & $29.38 \pm 1.35$ & $22.98 \pm 0.93$ \\
\hline $5 g$ & $2-\mathrm{F}$ & $6.01 \pm 0.90$ & $26.83 \pm 0.78$ & $>50$ & $>50$ \\
\hline $5 \mathrm{~h}$ & $\mathrm{H}$ & $>50$ & $>50$ & $>50$ & $>50$ \\
\hline $5 \mathbf{i}$ & $3-\mathrm{Cl}$ & $8.23 \pm 0.92$ & $30.16 \pm 0.90$ & $>50$ & $22.99 \pm 0.99$ \\
\hline $5 \mathbf{j}$ & $2-\mathrm{Cl}$ & $15.84 \pm 1.20$ & $34.06 \pm 1.23$ & $29.83 \pm 1.67$ & $30.09 \pm 1.32$ \\
\hline $5 \mathbf{k}$ & $3,4,5-\left(\mathrm{OCH}_{3}\right)_{3}$ & $33.09 \pm 1.12$ & $38.42 \pm 1.18$ & $>50$ & $>50$ \\
\hline 51 & $2-\mathrm{OCH}_{3}, 4-\mathrm{NO}_{2}$ & $32.50 \pm 1.51$ & $39.20 \pm 0.97$ & $35.07 \pm 1.65$ & $28.14 \pm 1.16$ \\
\hline $5 \mathrm{~m}$ & $\mathrm{Ph}$ & $7.89 \pm 0.90$ & $>50$ & $8.19 \pm 0.96$ & $>50$ \\
\hline $5 n$ & $4-\mathrm{Cl}$ & $21.81 \pm 1.40$ & $17.06 \pm 1.21$ & $>50$ & $24.72 \pm 1.27$ \\
\hline 50 & $3-\mathrm{Cl}, 4-\mathrm{F}$ & $>50$ & $38.17 \pm 0.82$ & $>50$ & $39.44 \pm 1.42$ \\
\hline $5 p$ & $3,4-(\mathrm{Cl})_{2}$ & $>50$ & $33.63 \pm 0.87$ & $>50$ & $19.99 \pm 1.06$ \\
\hline $5 q$ & $2-\mathrm{OCH}_{2} \mathrm{CH}_{3}$ & $22.14 \pm 1.35$ & $25.94 \pm 0.16$ & $17.03 \pm 1.23$ & $>50$ \\
\hline $5 r$ & $2-\mathrm{CH}_{3}$ & $25.53 \pm 1.21$ & $22.03 \pm 1.28$ & $>50$ & $>50$ \\
\hline $5-\mathbf{F} \mathbf{U}^{b}$ & & $14.78 \pm 0.66$ & $16.17 \pm 0.77$ & $10.27 \pm 0.59$ & $18.99 \pm 0.64$ \\
\hline
\end{tabular}

${ }^{a}$ Anti-tumor activity was assayed by exposure for $72 \mathrm{~h}$ to substances and expressed as concentration required to inhibit tumor cells proliferation by $50 \%$ (IC $\mathrm{C}_{50}$ ).

${ }^{b}$ Used as a positive control.

$\mathbf{5 i} 、 5 \mathbf{j}$ 等, 活性显著增强, 其中化合物 $\mathbf{5 g}$ 的 $\mathrm{IC}_{50}$ 达到了 $6.01 \mu \mathrm{mol} / \mathrm{L}$, 说明苯环上有单取代的卤素(氟、氯)时, 可 以显著地增强化合物的活性; 而当苯环上有双取代卤素 时，如化合物 5o、5p 时，活性明显消失，说明苯环上有 双取代的卤素时不利于生物活性的表达; 当将取代基换 为烷氧基和硝基多取代时, 如化合物 $\mathbf{5 k}$ 和 $\mathbf{5 l}$, 活性较 差, 说明多取代的烷氧基或硝基同样对化合物的活性提 升没有帮助. 总之, 当苯环上有单取代的烷基或卤素时, 有利于活性的增强.

\section{2 结论}

为了寻找高效低毒抗肿瘤化合物, 设计并制备出了 一系列新型 1,3-二取代酞嗪酮类衍生物, 利用核磁共振 氢谱、核磁共振碳谱和高分辨质谱等技术证实了目标化 合物的结构. 运用噻唑蓝(MTT)比色法测定了目标化合 物对人类四种癌细胞: MCF-7(人乳腺癌细胞)、PC-3(人 前列腺癌细胞) 、SW-620(人结肠癌细胞) 和 HGC-27(人胃 癌细胞) 四种人类癌细胞的抗增殖活性进行评价. 结果 显示大部分化合物具有较好的抗增殖活, 其中, 化合物 $5 \mathrm{~g}$ 对 $\mathrm{MCF}-7$ 细胞的抗增殖活性较好, $\mathrm{IC}_{50}$ 值为 6.01 $\mu \mathrm{mol} / \mathrm{L}$.

\section{3 实验部分}

\section{1 实验与仪器}

${ }^{1} \mathrm{H}$ NMR 和 ${ }^{13} \mathrm{C}$ NMR 光谱测定使用 DPX-DPX-400
超导核磁共振仪, TMS 作为内标; 高分辨质谱使用 Waters-Micromass 公司 Q-Tof 质谱仪测定; 硅胶: 中国 青岛海洋化工集团公司; 柱色谱硅胶: 上海五四化学试 剂厂; 邻苯二甲酸䣶: 天津化学试剂有限公司; 氯乙酰 氯: 天津永达化学试剂有限公司; $N, N$-二甲基甲酰胺: 广东光华科技有限公司; 无水乙醇: 烟台市化工有限公 司; 本实验分离纯化所用有机溶剂均为工业级, 经重新 蒸馏后使用, 其它试剂均为市售分析纯, 必要时做常规 处理.

\section{2 实验方法}

\subsubsection{2-(4-溴苯甲酰基)苯甲酸(2)的合成}

化合物 2 按照文献[23]的方法制备, 表征数据与文 献一致.

\subsubsection{4-(4-溴苯基)酞嗪-1(2H)-酮(3)的合成}

在室温条件下，将化合物 7 (18.3 g, $60 \mathrm{mmol})$ 溶于 $10 \%$ 的氢氧化钠水溶液中, 滴加 $80 \%$ 水合肼 $(3.72 \mathrm{~mL}$, $120 \mathrm{mmol}$ ), $110{ }^{\circ} \mathrm{C}$ 回流 $4 \mathrm{~h}$, 冷却至室温, 有大量固体析 出, 过滤, 干燥得到白色产物 $11.7 \mathrm{~g}$, 收率 65.5\%. m.p. $190 \sim 191{ }^{\circ} \mathrm{C} ;{ }^{1} \mathrm{H}$ NMR (400 MHz, DMSO- $d_{6}$ ) $\delta: 12.89$ (s, $1 \mathrm{H}), 8.35(\mathrm{dd}, J=6.1,3.1 \mathrm{~Hz}, 1 \mathrm{H}), 7.92 \sim 7.89(\mathrm{~m}, 2 \mathrm{H})$, $7.76(\mathrm{~d}, J=8.4 \mathrm{~Hz}, 2 \mathrm{H}), 7.69 \sim 7.66(\mathrm{~m}, 1 \mathrm{H}), 7.56(\mathrm{~d}, J=$ $8.4 \mathrm{~Hz}, 2 \mathrm{H}) ;{ }^{13} \mathrm{C}$ NMR $\left(101 \mathrm{MHz}\right.$, DMSO- $\left.d_{6}\right) \delta: 159.3$, $145.6,134.1,133.8,131.8,131.5,131.4,128.6,127.6$, 126.3, 126.1, 122.4; HRMS (ESI) calcd for $\mathrm{C}_{22} \mathrm{H}_{15} \mathrm{BrN}_{4-}$ 
$\mathrm{NaO}_{4}[\mathrm{M}+\mathrm{Na}]^{+}$322.9796, found 322.9795 .

\subsection{3 化合物 $\mathbf{4 a} \sim \mathbf{4 r}$ 的合成}

化合物 4a 按照文献[24]的方法制备, 表征数据与文 献一致.

化合物 $4 \mathrm{~b} 、 4 \mathrm{~d} 、 4 \mathrm{e} 、 4 \mathrm{f} 、 4 \mathrm{~h} 、 4 \mathrm{i} 、 4 \mathrm{j} 、 4 \mathrm{n} 、 4 \mathrm{r}$ 按照 文献[25]的方法制备, 表征数据与文献一致.

化合物 4c、4m、4o 按照文献[26]的方法制备，表征 数据与文献一致.

化合物 4k 按照文献[27]的方法制备, 表征数据与文 献一致.

化合物 $\mathbf{4 g}$ 按照文献[28]的方法制备, 表征数据与文 献一致。

化合物 $4 p$ 按照文献[29]的方法制备, 表征数据与文 献一致.

化合物 4q 按照文献[30]的方法制备, 表征数据与文 献一致。

化合物 41 按照文献[31]的方法制备, 表征数据与文 献一致.

\subsection{4 目标化合物 $\mathbf{5 a} \sim \mathbf{5 r}$ 的合成}

2-(4-(4-溴苯基)-1-氧亚基唒嗪-2(1H)-基)- $\mathrm{N}$-(2-乙基 苯基)乙酰胺 $(\mathbf{5 a})$ : 在室温条件下, 将化合物 $3(1 \mathrm{mmol})$ 用 $10 \mathrm{~mL}$ 丙酮溶解, 加入缚酸剂碳酸钾 $(1.5 \mathrm{mmol})$, 将 化合物 $4 \mathrm{a}(1.2 \mathrm{mmol})$ 用 $5 \mathrm{~mL}$ 丙酮溶解, 滴加至上反应 液中, $60{ }^{\circ} \mathrm{C}$ 回流反应 $6 \mathrm{~h}$, 冷却至室温, 抽滤, 少量丙酮 洗涤, 滤饼加入冰水中搅拌 $1 \mathrm{~h}$, 抽滤, 干燥, 得到白色 产物 5a, 收率 78.3\%. m.p. 267 268 ${ }^{\circ} \mathrm{C} ;{ }^{1} \mathrm{H}$ NMR (400 MHz, DMSO- $\left.d_{6}\right) \delta: 9.60(\mathrm{~s}, 1 \mathrm{H}), 8.39(\mathrm{~s}, 1 \mathrm{H}), 7.93$ (d, $J=$ $2.8 \mathrm{~Hz}, 2 \mathrm{H}), 7.78(\mathrm{~d}, J=7.7 \mathrm{~Hz}, 2 \mathrm{H}), 7.72(\mathrm{~s}, 1 \mathrm{H}), 7.58(\mathrm{~d}$, $J=7.7 \mathrm{~Hz}, 2 \mathrm{H}), 7.34$ (d, $J=5.7 \mathrm{~Hz}, 1 \mathrm{H}), 7.23(\mathrm{~s}, 1 \mathrm{H}), 7.16$ (s, 2H), 5.06 (s, 2H), 2.61 (d, $J=7.3 \mathrm{~Hz}, 2 \mathrm{H}), 1.11$ (t, $J=$ $7.1 \mathrm{~Hz}, 3 \mathrm{H}) ;{ }^{13} \mathrm{C}$ NMR (101 MHz, DMSO- $\left.d_{6}\right) \delta: 165.9$, $158.3,145.2,138.1,135.0,133.8,133.7,132.0,131.7$, $131.5,131.4,128.5,127.4,127.0,126.5,126.4,125.9$, 125.9, 122.6, 54.0, 23.7, 14.2; HRMS (ESI) calcd for $\mathrm{C}_{24} \mathrm{H}_{20} \mathrm{BrN}_{3} \mathrm{NaO}_{2}[\mathrm{M}+\mathrm{Na}]^{+}$484.0637, found 484.0636.

2-(4-(4-溴苯基)-1-氧亚基酞嗪-2(1H)-基)- $N$-(2-硝基 苯基)乙酰胺 $(5 \mathrm{~b})$ : 将 $4 \mathrm{a}$ 换成 $\mathbf{4 b}$, 操作和投料比同化合 物 5a 的制备, 得到黄色产物 5b, 收率 78.6\%. m.p. 174 $175^{\circ} \mathrm{C} ;{ }^{1} \mathrm{H}$ NMR (400 MHz, DMSO- $\left.d_{6}\right) \delta: 10.60(\mathrm{~s}, 1 \mathrm{H})$, $8.48 \sim 8.29(\mathrm{~m}, 1 \mathrm{H}), 8.02 \sim 7.90(\mathrm{~m}, 3 \mathrm{H}), 7.82 \sim 7.69(\mathrm{~m}$, $5 \mathrm{H}$ ), $7.62 \sim 7.56(\mathrm{~m}, 2 \mathrm{H}), 7.39$ (ddd, $J=8.5,7.2,1.6 \mathrm{~Hz}$, $1 \mathrm{H}), 5.10(\mathrm{~s}, 2 \mathrm{H}) ;{ }^{13} \mathrm{C}$ NMR (101 MHz, DMSO- $\left.d_{6}\right) \delta$ : $166.1,158.3,145.5,141.9,134.1,133.8,133.6,132.1$, $131.6,131.5,130.8,128.4,127.3,126.7,126.5,125.4$, 125.1, 125.0, 122.7, 54.1; HRMS (ESI) calcd for
$\mathrm{C}_{22} \mathrm{H}_{15} \mathrm{BrN}_{4} \mathrm{NaO}_{4}[\mathrm{M}+\mathrm{Na}]^{+}$501.0174, found 501.0174.

2-(4-(4-溴苯基)-1-氧亚基唒嗪-2(1H)-基)- $\mathrm{N}$-(3-氟苯 基)乙酰胺 $(\mathbf{5 c})$ : 将 $\mathbf{4 a}$ 换成 $\mathbf{4 c}$, 操作和投料比同化合物 5a 的制备, 得到白色产物 5c, 收率 61.3\%. m.p. 262 $263{ }^{\circ} \mathrm{C} ;{ }^{1} \mathrm{H}$ NMR (400 MHz, DMSO- $\left.d_{6}\right) \delta: 10.55(\mathrm{~s}, 1 \mathrm{H})$, $8.44 \sim 8.31(\mathrm{~m}, 1 \mathrm{H}), 8.01 \sim 7.89(\mathrm{~m}, 2 \mathrm{H}), 7.83 \sim 7.70(\mathrm{~m}$, $3 \mathrm{H}), 7.64 \sim 7.54(\mathrm{~m}, 3 \mathrm{H}), 7.44 \sim 7.27(\mathrm{~m}, 2 \mathrm{H}), 6.90(\mathrm{ddt}$, $J=8.6,7.3,1.2 \mathrm{~Hz}, 1 \mathrm{H}), 5.07$ (s, 2H); ${ }^{13} \mathrm{C}$ NMR $(101 \mathrm{MHz}$, DMSO- $\left.d_{6}\right) \delta: 165.8,163.3(\mathrm{~d}, J=242.4 \mathrm{~Hz}), 158.3,145.2$, $140.4,133.8,133.7,132.1,131.6,131.4,130.4$ (d, $J=10.1$ $\mathrm{Hz}), 128.4,127.3,126.5,122.7,114.9,110.0(\mathrm{~d}, J=20.2$ $\mathrm{Hz}$ ), 106.1, 105.8, 54.4; HRMS (ESI) calcd for $\mathrm{C}_{22} \mathrm{H}_{15} \mathrm{Br}-$ $\mathrm{FN}_{3} \mathrm{NaO}_{2}[\mathrm{M}+\mathrm{Na}]^{+}$474.0229, found 474.0221.

2-(4-(4-溴苯基)-1-氧亚基酞嗪-2(1H)-基)- $N$-(4-硝基 苯基)乙酰胺 $(\mathbf{5 d})$ : 将 $\mathbf{4 a}$ 换成 $\mathbf{4 d}$, 操作和投料比同化合 物 5a 的制备, 得黄色产物 5d, 收率 65.1\%. m.p. 285 $286{ }^{\circ} \mathrm{C} ;{ }^{1} \mathrm{H}$ NMR (400 MHz, DMSO- $\left.d_{6}\right) \delta: 10.96$ (s, $\left.1 \mathrm{H}\right)$, $8.44 \sim 8.33(\mathrm{~m}, 1 \mathrm{H}), 8.28 \sim 8.20(\mathrm{~m}, 2 \mathrm{H}), 8.00 \sim 7.91(\mathrm{~m}$, 2H), $7.89 \sim 7.81(\mathrm{~m}, 2 \mathrm{H}), 7.81 \sim 7.71(\mathrm{~m}, 3 \mathrm{H}), 7.64 \sim 7.55$ (m, 2H), $5.12(\mathrm{~s}, 2 \mathrm{H}) ;{ }^{13} \mathrm{C}$ NMR $\left(101 \mathrm{MHz}\right.$, DMSO- $\left.d_{6}\right) \delta$ : $166.5,158.3,145.3,144.7,142.3,133.8,133.6,132.1$, $131.6,131.4,128.4,127.2,126.5,126.5,125.0,122.7$, 118.9, 54.6; HRMS (ESI) calcd for $\mathrm{C}_{22} \mathrm{H}_{15} \mathrm{BrN}_{4} \mathrm{NaO}_{4}[\mathrm{M}+$ $\mathrm{Na}]^{+}$501.0174, found 501.0176.

2-(4-(4-溴苯基)-1-氧亚基酞嗪-2(1H)-基)- $N$-(3-甲基 苯基)乙酰胺 (5e): 将 $4 \mathrm{a}$ 换成 $4 \mathrm{e}$, 操作和投料比同化合物 5a 的制备, 得到白色产物 5e, 收率 56.7\%. m.p. 265 $266{ }^{\circ} \mathrm{C} ;{ }^{1} \mathrm{H}$ NMR (400 MHz, DMSO-d $\left.\mathrm{d}_{6}\right) \delta: 10.23(\mathrm{~s}, 1 \mathrm{H})$, $8.45 \sim 8.30(\mathrm{~m}, 1 \mathrm{H}), 7.98 \sim 7.90(\mathrm{~m}, 2 \mathrm{H}), 7.80 \sim 7.71(\mathrm{~m}$, $3 \mathrm{H}), 7.62 \sim 7.55(\mathrm{~m}, 2 \mathrm{H}), 7.44(\mathrm{~s}, 1 \mathrm{H}), 7.40 \sim 7.34(\mathrm{~m}$, $1 \mathrm{H}), 7.19$ (t, $J=7.8 \mathrm{~Hz}, 1 \mathrm{H}), 6.88(\mathrm{~d}, J=7.5 \mathrm{~Hz}, 1 \mathrm{H}), 5.03$ (s, 2H), $2.27(\mathrm{~s}, 3 \mathrm{H}) ;{ }^{13} \mathrm{C}$ NMR (101 MHz, DMSO- $\left.d_{6}\right) \delta$ : $165.3,158.3,145.1,138.6,137.9,133.7,132.0,131.6$, $131.4,128.5,128.4,127.3,126.5,126.4,124.1,122.7$, 119.7, 116.3, 54.3, 21.1; HRMS(ESI) calcd for $\mathrm{C}_{23} \mathrm{H}_{18} \mathrm{Br}-$ $\mathrm{N}_{3} \mathrm{NaO}_{2}[\mathrm{M}+\mathrm{Na}]^{+}$470.0480, found 470.0482 .

2-(4-(4-溴苯基)-1-氧亚基酞嗪-2(1H)-基)- $N$-(3-硝基 苯基)乙酰胺 $(\mathbf{5 f})$ : 将 $\mathbf{4 a}$ 换成 $\mathbf{4 f}$, 操作和投料比同化合物 5a 的制备, 得到黄色产物 5f, 收率 50.8\%. m.p. 239 $240{ }^{\circ} \mathrm{C} ;{ }^{1} \mathrm{H}$ NMR (400 MHz, DMSO- $\left.d_{6}\right) \delta: 10.83(\mathrm{~s}, 1 \mathrm{H})$, $8.62(\mathrm{t}, J=2.2 \mathrm{~Hz}, 1 \mathrm{H}), 8.46 \sim 8.33(\mathrm{~m}, 1 \mathrm{H}), 7.94$ (dddd, $J=9.9,7.8,5.6,3.4 \mathrm{~Hz}, 4 \mathrm{H}), 7.82 \sim 7.71(\mathrm{~m}, 3 \mathrm{H}), 7.67 \sim$ $7.56(\mathrm{~m}, 3 \mathrm{H}), 5.10(\mathrm{~s}, 2 \mathrm{H}) ;{ }^{13} \mathrm{C}$ NMR (101MHz, DMSO$\left.d_{6}\right) \delta: 166.3,158.3,147.9,145.3,139.7,133.8,133.6$, $132.1,131.6,131.4,130.2,128.4,127.3,126.5,126.5$, 
125.1, 122.7, 118.0, 113.3, 54.5; HRMS (ESI) calcd for $\mathrm{C}_{22} \mathrm{H}_{15} \mathrm{BrN}_{4} \mathrm{NaO}_{4}[\mathrm{M}+\mathrm{Na}]^{+}$501.0174, found 501.0174.

2-(4-(4-溴苯基)-1-氧亚基酞嗪-2 (1H)-基)- $N$-(2-氟苯 基)乙酰胺 $(5 \mathrm{~g})$ : 将 $4 \mathrm{a}$ 换成 $4 \mathrm{~g}$, 操作和投料比同化合物 5a 的制备, 得到白色产物 5g, 收率 51.4\%. m.p. 261 $262{ }^{\circ} \mathrm{C} ;{ }^{1} \mathrm{H}$ NMR (400 MHz, DMSO- $\left.d_{6}\right) \delta: 10.15(\mathrm{~s}, 1 \mathrm{H})$, $8.39(\mathrm{dd}, J=6.3,3.1 \mathrm{~Hz}, 1 \mathrm{H}), 7.98 \sim 7.86(\mathrm{~m}, 3 \mathrm{H}), 7.82 \sim$ $7.69(\mathrm{~m}, 3 \mathrm{H}), 7.62 \sim 7.53(\mathrm{~m}, 2 \mathrm{H}), 7.32 \sim 7.23(\mathrm{~m}, 1 \mathrm{H})$, 7.16 (tq, $J=7.3,4.1,3.3 \mathrm{~Hz}, 2 \mathrm{H}), 5.12(\mathrm{~s}, 2 \mathrm{H}) ;{ }^{13} \mathrm{C}$ NMR $\left(101 \mathrm{MHz}\right.$, DMSO- $\left.d_{6}\right) \delta: 166.0,158.3,154.6(\mathrm{~d}, J=242.4$ $\mathrm{Hz}), 145.1,133.7$ (d, $J=10.1 \mathrm{~Hz}), 132.0,131.6,131.4$, $128.4,127.3,126.5,125.7,125.6,125.4,124.4,124.3(\mathrm{~d}$, $J=3.0 \mathrm{~Hz}), 123.8,122.7,115.6(\mathrm{~d}, J=20.2 \mathrm{~Hz}), 54.1$; HRMS (ESI) calcd for $\mathrm{C}_{22} \mathrm{H}_{15} \mathrm{BrFN}_{3} \mathrm{NaO}_{2}[\mathrm{M}+\mathrm{Na}]^{+}$ 474.0229, found 474.0231.

2-(4-(4-溴苯基)-1-氧亚基酞嗪-2(1H)-基)- $\mathrm{N}$-苯基乙 酰胺 $(5 \mathrm{~h})$ : 将 $4 \mathrm{a}$ 换成 $4 \mathrm{~h}$, 操作和投料比同化合物 $5 \mathrm{a}$ 的 制备, 得到白色产物 $\mathbf{5 h}$, 收率 $68.3 \%$. m.p. $>300{ }^{\circ} \mathrm{C} ;{ }^{1} \mathrm{H}$ NMR (400 MHz, DMSO- $\left.d_{6}\right) \delta: 10.36(\mathrm{~s}, 1 \mathrm{H}), 8.45 \sim 8.31$ (m, $1 \mathrm{H}), 8.03 \sim 7.88(\mathrm{~m}, 2 \mathrm{H}), 7.81 \sim 7.70(\mathrm{~m}, 3 \mathrm{H}), 7.59$ (dd, $J=8.0,5.6 \mathrm{~Hz}, 4 \mathrm{H}), 7.32(\mathrm{t}, J=7.8 \mathrm{~Hz}, 2 \mathrm{H}), 7.06(\mathrm{t}$, $J=7.4 \mathrm{~Hz}, 1 \mathrm{H}), 5.05(\mathrm{~s}, 2 \mathrm{H}) ;{ }^{13} \mathrm{C}$ NMR $(101 \mathrm{MHz}$, DMSO- $\left.d_{6}\right) \delta: 165.4,159.1,158.3,145.3,138.6,134.2$, $133.6,131.7,131.6,131.5,131.4,128.7,127.8,126.3$, 126.1, 122.4, 119.1, 54.3; HRMS (ESI) calcd for $\mathrm{C}_{22} \mathrm{H}_{16} \mathrm{BrN}_{3} \mathrm{NaO}_{2}[\mathrm{M}+\mathrm{Na}]^{+}$456.0324, found 456.0325.

2-(4-(4-溴苯基)-1-氧亚基唒嗪-2 $(1 H)$-基)- $N$-(3-氯苯 基)乙酰胺 $(\mathbf{5 i})$ : 将 $\mathbf{4 a}$ 换成 $\mathbf{4 i}$, 操作和投料比同化合物 $\mathbf{5 a}$ 的制备, 得到白色产物 $\mathbf{5 i}$, 收率 $71.9 \%$. m.p. 251 $252{ }^{\circ} \mathrm{C} ;{ }^{1} \mathrm{H}$ NMR (400 MHz, DMSO- $d_{6}$ ) $\delta: 10.33(\mathrm{~s}, 1 \mathrm{H})$, $8.42(\mathrm{dd}, J=5.8,3.2 \mathrm{~Hz}, 1 \mathrm{H}), 8.18 \sim 8.14(\mathrm{~m}, 1 \mathrm{H}), 7.94$ (dd, $J=5.7,3.1 \mathrm{~Hz}, 2 \mathrm{H}), 7.78(\mathrm{t}, J=8.3 \mathrm{~Hz}, 3 \mathrm{H}), 7.73(\mathrm{dd}$, $J=5.8,3.1 \mathrm{~Hz}, 1 \mathrm{H}), 7.67(\mathrm{~d}, J=7.3 \mathrm{~Hz}, 1 \mathrm{H}), 7.60$ (d, $J=$ $8.3 \mathrm{~Hz}, 1 \mathrm{H}), 7.54$ (d, $J=2.8 \mathrm{~Hz}, 1 \mathrm{H}), 7.49$ (t, $J=7.8 \mathrm{~Hz}$, $1 \mathrm{H}), 5.21(\mathrm{~s}, 2 \mathrm{H}) ;{ }^{13} \mathrm{C}$ NMR $\left(101 \mathrm{MHz}, \mathrm{DMSO}-d_{6}\right) \delta$ : 166.4, 158.4, 145.2, 133.8, 133.6, 133.3, 132.0, 131.6, $131.5,128.5,128.0,127.5,126.5,126.0,125.7,125.5$, 122.9, 122.7, 121.8, 54.3; HRMS (ESI) calcd for $\mathrm{C}_{22} \mathrm{H}_{15^{-}}$ $\mathrm{BrClN}_{3} \mathrm{NaO}_{2}[\mathrm{M}+\mathrm{Na}]^{+}$489.9934, found 489.9935 .

2-(4-(4-溴苯基)-1-氧亚基唒嗪-2(1H)-基)- $N$-(2-氯苯 基)乙酰胺 $(\mathbf{5 j})$ : 将 $\mathbf{4 a}$ 换成 $\mathbf{4 j}$, 操作和投料比同化合物 $\mathbf{5 a}$ 的制备, 得到白色产物 $\mathbf{5 j}$, 收率 75.3\%. m.p. 250 $251{ }^{\circ} \mathrm{C} ;{ }^{1} \mathrm{H}$ NMR (400 MHz, DMSO- $\left.d_{6}\right) \delta: 9.89$ (s, $\left.1 \mathrm{H}\right)$, 8.37 (ddd, $J=14.9,7.5,3.4 \mathrm{~Hz}, 1 \mathrm{H}), 7.98 \sim 7.90(\mathrm{~m}, 2 \mathrm{H})$, $7.76(\mathrm{dd}, J=15.4,7.8 \mathrm{~Hz}, 4 \mathrm{H}), 7.59$ (d, $J=8.4 \mathrm{~Hz}, 2 \mathrm{H})$, $7.51(\mathrm{dd}, J=8.0,1.4 \mathrm{~Hz}, 1 \mathrm{H}), 7.37 \sim 7.27(\mathrm{~m}, 1 \mathrm{H}), 7.20$ $(\mathrm{td}, J=7.8,1.7 \mathrm{~Hz}, 1 \mathrm{H}), 5.12(\mathrm{~s}, 2 \mathrm{H}) ;{ }^{13} \mathrm{C}$ NMR $(101 \mathrm{MHz}$, DMSO- $\left.d_{6}\right) \delta: 166.0,158.3,145.2,134.4,133.7,133.7$, $132.1,131.6,131.5,131.4,129.5,128.4,127.4,127.3$, 126.5, 126.5, 122.7, 121.9, 102.7, 54.1; HRMS (ESI) calcd for $\mathrm{C}_{22} \mathrm{H}_{15} \mathrm{BrClN}_{3} \mathrm{NaO}_{2}[\mathrm{M}+\mathrm{Na}]^{+}$489.9934, found 489.9931 .

2-(4-(4-溴苯基)-1-氧亚基酞嗪-2(1H)-基)- $N$-(3,4,5三甲氧基苯基)乙酰胺 $(5 \mathbf{k})$ : 将 $\mathbf{4 a}$ 换成 $\mathbf{4 k}$, 操作和投料 比同化合物 5a 的制备, 得到白色产物 $5 \mathbf{k}$, 收率 $57.1 \%$. m.p. $246 \sim 247{ }^{\circ} \mathrm{C} ;{ }^{1} \mathrm{H}$ NMR (400 MHz, DMSO- $\left.d_{6}\right) \delta$ : $10.29(\mathrm{~s}, 1 \mathrm{H}), 8.48 \sim 8.33(\mathrm{~m}, 1 \mathrm{H}), 7.99 \sim 7.89(\mathrm{~m}, 2 \mathrm{H})$, $7.82 \sim 7.70(\mathrm{~m}, 3 \mathrm{H}), 7.59$ (d, $J=8.4 \mathrm{~Hz}, 2 \mathrm{H}), 6.98(\mathrm{~s}, 2 \mathrm{H})$, $5.02(\mathrm{~s}, 2 \mathrm{H}), 3.73(\mathrm{~s}, 6 \mathrm{H}), 3.62(\mathrm{~s}, 3 \mathrm{H}) ;{ }^{13} \mathrm{C}$ NMR $(101$ MHz, DMSO- $\left.d_{6}\right) \delta: 165.3,158.3,152.7,145.1,134.8$, 133.7, 133.7, 133.5, 132.1, 132.1, 131.6, 131.4, 128.4, 127.3, 126.5, 122.7, 96.8, 60.0, 55.6, 54.3; HRMS (ESI) calcd for $\mathrm{C}_{25} \mathrm{H}_{22} \mathrm{BrN}_{3} \mathrm{NaO}_{5}[\mathrm{M}+\mathrm{Na}]^{+}$546.0641, found 546.0643 .

2-(4-(4-溴苯基)-1-氧亚基酞嗪-2(1H)-基)- $N$-(2-甲氧 基-4-硝基苯基)乙酰胺 (5I): 将 $4 \mathrm{a}$ 换成 $4 \mathrm{l}$, 操作和投料比 同化合物 5a 的制备, 得到白色产物 51, 收率 49.5\%. m.p. $249 \sim 250{ }^{\circ} \mathrm{C} ;{ }^{1} \mathrm{H}$ NMR $\left(400 \mathrm{MHz}\right.$, DMSO- $\left.d_{6}\right) \delta: 10.11$ (s, $1 \mathrm{H}), 8.41 \sim 8.32(\mathrm{~m}, 2 \mathrm{H}), 7.98 \sim 7.91(\mathrm{~m}, 2 \mathrm{H}), 7.89 \sim 7.82$ $(\mathrm{m}, 2 \mathrm{H}), 7.80 \sim 7.70(\mathrm{~m}, 3 \mathrm{H}), 7.59(\mathrm{~d}, J=8.4 \mathrm{~Hz}, 2 \mathrm{H})$, $5.22(\mathrm{~s}, 2 \mathrm{H}), 4.02(\mathrm{~s}, 3 \mathrm{H}) ;{ }^{13} \mathrm{C}$ NMR $\left(101 \mathrm{MHz}\right.$, DMSO-d $\left.d_{6}\right)$ $\delta: 166.7,158.3,148.6,145.2,142.8,133.9,133.8,133.6$, $132.1,131.6,131.4,128.4,127.2,126.5,126.5,122.7$, 119.5, 116.7, 106.0, 56.5, 54.7; HRMS (ESI) calcd for $\mathrm{C}_{23} \mathrm{H}_{17} \mathrm{BrN}_{4} \mathrm{NaO}_{5}[\mathrm{M}+\mathrm{Na}]^{+}$531.0280, found 531.0287.

2-(4-(4-溴苯基)-1-氧亚基酞嗪-2(1H)-基)- $N$-(萗-1基)乙酰胺 $(5 \mathrm{~m})$ : 将 $\mathbf{4 a}$ 换成 $\mathbf{4 m}$, 操作和投料比同化合物 5a 的制备, 得白色产物 5m, 收率 75.2\%. m.p. 287 $288{ }^{\circ} \mathrm{C} ;{ }^{1} \mathrm{H}$ NMR (400 MHz, DMSO- $\left.d_{6}\right) \delta$ : $10.25(\mathrm{~s}, 1 \mathrm{H})$, $8.42(\mathrm{dt}, J=7.3,3.7 \mathrm{~Hz}, 1 \mathrm{H}), 8.14(\mathrm{dt}, J=7.0,3.5 \mathrm{~Hz}, 1 \mathrm{H})$, $7.94(\mathrm{dt}, J=7.2,3.6 \mathrm{~Hz}, 3 \mathrm{H}), 7.79(\mathrm{dd}, J=8.5,4.2 \mathrm{~Hz}$, $3 \mathrm{H}), 7.73(\mathrm{dt}, J=6.1,3.6 \mathrm{~Hz}, 1 \mathrm{H}), 7.67(\mathrm{~d}, J=7.4 \mathrm{~Hz}$, $1 \mathrm{H}), 7.60(\mathrm{~d}, J=8.2 \mathrm{~Hz}, 2 \mathrm{H}), 7.57 \sim 7.45(\mathrm{~m}, 3 \mathrm{H}), 5.21(\mathrm{~s}$, $2 \mathrm{H}) ;{ }^{13} \mathrm{C}$ NMR (101 MHz, DMSO- $\left.d_{6}\right) \delta$ : 166.4, 158.4, $145.3,133.8,133.7,133.6,133.1,132.0,131.6,131.5$, $128.5,128.0,127.9,127.5,126.5,126.4,126.0,125.8$, 125.6, 125.5, 122.8, 122.7, 121.9, 54.3; HRMS (ESI) calcd for $\mathrm{C}_{26} \mathrm{H}_{18} \mathrm{BrN}_{3} \mathrm{NaO}_{2}[\mathrm{M}+\mathrm{Na}]^{+}$506.0840, found 506.0842 .

2-(4-(4-溴苯基)-1-氧亚基酞嗪-2(1H)-基)- $\mathrm{N}$-(4-氯苯 
基)乙酰胺(5n): 将 $4 \mathrm{a}$ 换成 $\mathbf{4 n}$, 操作和投料比同化合物 5a 的制备, 得到白色产物 5n, 收率 58.9\%. m.p. 273 $274{ }^{\circ} \mathrm{C} ;{ }^{1} \mathrm{H}$ NMR (400 MHz, DMSO- $\left.d_{6}\right) \delta: 10.48(\mathrm{~s}, 1 \mathrm{H})$, $8.54 \sim 8.24(\mathrm{~m}, 1 \mathrm{H}), 8.04 \sim 7.87(\mathrm{~m}, 2 \mathrm{H}), 7.82 \sim 7.54(\mathrm{~m}$, $7 \mathrm{H}), 7.42 \sim 7.33(\mathrm{~m}, 2 \mathrm{H}), 5.04(\mathrm{~s}, 2 \mathrm{H}) ;{ }^{13} \mathrm{C}$ NMR $(101$ $\left.\mathrm{MHz}, \mathrm{DMSO}-d_{6}\right) \delta$ : $165.6,158.3,145.2,137.5,133.8$, $133.6,132.1,131.6,131.5,129.3,128.7,128.4,127.3$, 127.0, 126.5, 122.7, 120.7, 54.4; HRMS (ESI) calcd for $\mathrm{C}_{22} \mathrm{H}_{15} \mathrm{BrClN}_{3} \mathrm{NaO}_{2}[\mathrm{M}+\mathrm{Na}]^{+}$489.9934, found 489.9935 .

2-(4-(4-溴苯基)-1-氧亚基酞嗪-2(1H)-基)- $N$-(3-氯-4氟苯基)乙酰胺 (5o): 将 $\mathbf{4 a}$ 换成 $\mathbf{4 o}$, 操作和投料比同化 合物 5a 的制备, 得到白色产物 5o, 收率 $48.9 \%$. m.p. $274 \sim 275{ }^{\circ} \mathrm{C} ;{ }^{1} \mathrm{H}$ NMR (400 MHz, DMSO- $d_{6}$ ) $\delta$ : 10.54 (s, $1 \mathrm{H}), 8.42 \sim 8.34(\mathrm{~m}, 1 \mathrm{H}), 7.99 \sim 7.86(\mathrm{~m}, 3 \mathrm{H}), 7.82 \sim 7.71$ (m, 3H), $7.62 \sim 7.55(\mathrm{~m}, 2 \mathrm{H}), 7.48$ (ddd, $J=9.0,4.4,2.6$ $\mathrm{Hz}, 1 \mathrm{H}), 7.39$ (t, $J=9.1 \mathrm{~Hz}, 1 \mathrm{H}), 5.04(\mathrm{~s}, 2 \mathrm{H}) ;{ }^{13} \mathrm{C} \mathrm{NMR}$ (101 MHz, DMSO- $\left.d_{6}\right) \delta: 165.8,158.3,154.4(\mathrm{~d}, J=252.5$ $\mathrm{Hz}), 145.2,135.8$ (d, $J=3.0 \mathrm{~Hz}), 133.8,133.6,132.1$, 131.6, 131.4, 128.4, 127.3, 126.5 (d, $J=2.0 \mathrm{~Hz}), 122.7$, 120.6, 119.5, 119.2 (d, $J=20.2 \mathrm{~Hz}), 117.1,116.9,54.3$; HRMS (ESI) calcd for $\mathrm{C}_{22} \mathrm{H}_{14} \mathrm{BrClFN}_{3} \mathrm{NaO}_{2}[\mathrm{M}+\mathrm{Na}]^{+}$ 507.9840, found 507.9842 .

2-(4-(4-澳苯基)-1-氧亚基酞嗪-2(1H)-基)- $N$-(3,4-二 氯苯基)乙酰胺 $(\mathbf{5 p})$ : 将 $\mathbf{4 a}$ 换成 $\mathbf{4 p}$, 操作和投料比同化 合物 5a 的制备, 得到白色产物 5p, 收率 61.2\%. m.p. $259 \sim 260{ }^{\circ} \mathrm{C} ;{ }^{1} \mathrm{H}$ NMR $\left(400 \mathrm{MHz}, \mathrm{DMSO}-d_{6}\right) \delta: 10.64$ (s, $1 \mathrm{H}), 8.42 \sim 8.33(\mathrm{~m}, 1 \mathrm{H}), 8.01 \sim 7.91(\mathrm{~m}, 3 \mathrm{H}), 7.76(\mathrm{dd}$, $J=12.5,6.0 \mathrm{~Hz}, 3 \mathrm{H}), 7.58(\mathrm{~d}, J=8.4 \mathrm{~Hz}, 3 \mathrm{H}), 7.49$ (dd, $J=8.8,2.4 \mathrm{~Hz}, 1 \mathrm{H}), 5.06$ (s, 2H); ${ }^{13} \mathrm{C}$ NMR $(101 \mathrm{MHz}$, DMSO- $\left.d_{6}\right) \delta: 166.0,158.3,145.2,138.7,133.8,133.6$, $132.1,131.6,131.4,131.0,130.7,128.4,127.3,126.5$, 126.5, 124.9, 122.7, 120.4, 119.2, 54.4; HRMS (ESI) calcd for $\mathrm{C}_{22} \mathrm{H}_{14} \mathrm{BrCl}_{2} \mathrm{~N}_{3} \mathrm{NaO}_{2}[\mathrm{M}+\mathrm{Na}]^{+}$523.9544, found 523.9546.

2-(4-(4-溴苯基)-1-氧亚基酞嗪-2(1H)-基)- $N$-(2-乙氧 基苯基)乙酰胺 (5q): 将 $\mathbf{4 a}$ 换成 $\mathbf{4 q}$, 操作和投料比同化 合物 $\mathbf{5 a}$ 的制备, 得到白色产物 $\mathbf{5 q}$, 收率 71.5\%. m.p. $229 \sim 230{ }^{\circ} \mathrm{C} ;{ }^{1} \mathrm{H}$ NMR (400 MHz, DMSO- $\left.d_{6}\right) \delta: 9.33$ (s, $1 \mathrm{H}), 8.44 \sim 8.35(\mathrm{~m}, 1 \mathrm{H}), 8.00 \sim 7.90(\mathrm{~m}, 3 \mathrm{H}), 7.81 \sim 7.69$ (m, 3H), $7.63 \sim 7.53(\mathrm{~m}, 2 \mathrm{H}), 7.09 \sim 6.99(\mathrm{~m}, 2 \mathrm{H}), 6.88$ (ddd, $J=8.4,6.6,2.2 \mathrm{~Hz}, 1 \mathrm{H}$ ), 5.13 (s, $2 \mathrm{H}$ ), $4.16 \sim 4.01$ $(\mathrm{m}, 2 \mathrm{H}), 1.31(\mathrm{t}, J=7.0 \mathrm{~Hz}, 3 \mathrm{H}) ;{ }^{13} \mathrm{C}$ NMR $(101 \mathrm{MHz}$, DMSO- $d_{6}$ ) $\delta: 165.5,158.3,148.4,145.3,133.8,132.1$, $131.6,131.5,128.4,127.3,127.0,126.5,124.5,122.7$, 121.4, 120.2, 112.2, 63.9, 54.5, 14.4; HRMS (ESI) calcd for $\mathrm{C}_{24} \mathrm{H}_{20} \mathrm{BrN}_{3} \mathrm{NaO}_{3}[\mathrm{M}+\mathrm{Na}]^{+}$500.0586, found 500.0587.

2-(4-(4-澳苯基)-1-氧亚基唒嗪-2(1H)-基)- $\mathrm{N}$-(2-甲基 苯基)乙酰胺 $(\mathbf{5 r})$ : 将 $\mathbf{4 a}$ 换成 $\mathbf{4 r}$, 操作和投料比同化合物 5a 的制备, 得到白色产物 $5 \mathbf{r}$, 收率 49.9\%. m.p. 260 $261{ }^{\circ} \mathrm{C}$; ${ }^{1} \mathrm{H}$ NMR (400 MHz, DMSO- $\left.d_{6}\right) \delta: 9.64(\mathrm{~s}, 1 \mathrm{H})$, $8.45 \sim 8.36(\mathrm{~m}, 1 \mathrm{H}), 8.00 \sim 7.91(\mathrm{~m}, 2 \mathrm{H}), 7.78(\mathrm{~d}, J=8.6$ $\mathrm{Hz}, 2 \mathrm{H}), 7.57$ (t, $J=8.4 \mathrm{~Hz}, 3 \mathrm{H}), 7.40(\mathrm{~d}, J=7.8 \mathrm{~Hz}, 1 \mathrm{H})$, 7.15 (ddd, $J=34.9,17.6,7.4 \mathrm{~Hz}, 3 \mathrm{H}$ ), 5.08 (s, 2H), 2.23 (s, $3 \mathrm{H}) ;{ }^{13} \mathrm{C}$ NMR (101 MHz, DMSO- $\left.d_{6}\right) \delta$ : 165.6, 159.1, $158.3,145.1,135.8,133.7,132.0,131.6,131.5,131.4$, $130.3,128.5,127.4,126.5,126.4,125.9,125.3,125.0$, 122.6, 54.1, 17.8; HRMS (ESI) calcd for $\mathrm{C}_{23} \mathrm{H}_{18} \mathrm{BrN}_{3} \mathrm{NaO}_{2}$ $[\mathrm{M}+\mathrm{Na}]^{+} 470.0480$, found 470.0482 .

\subsection{5 抗增殖细胞活性实验}

称取 3 $5 \mathrm{mg}$ 样品置于 $1.5 \mathrm{~mL} \mathrm{EP}$ 管中, 用二甲基 亚砜(DMSO)配制成 $100 \times 10^{3} \mu \mathrm{g} / \mathrm{mL}$ 的溶液, $4{ }^{\circ} \mathrm{C}$ 保存, 根据实验所需浓度用培养基稀释. 取对数生长期的细 胞, 消化计数后, 用培养基调整细胞密度, 以 $4 \times 10^{3}$ 个/ 孔接种至 96 孔板，每孔 $150 \mu \mathrm{L}$ 培养基，培养 $24 \mathrm{~h}$, 弃去 培养基, 加入用培养基稀释好的药物, 即将待测样品以 $100,50.0,25.0,12.5,6.25,3.13,1.56,0.780 \mu \mathrm{g} / \mathrm{mL}$ 浓度 加入 96 孔板中, 每个浓度设 6 个复孔, 另设空白对照组 和阴性对照组. 药物作用 $72 \mathrm{~h}$, 每孔加入 $20 \mu \mathrm{L}$ 噻坐蓝 (MTT)溶液 $(5 \mathrm{mg} / \mathrm{mL}), 37{ }^{\circ} \mathrm{C}$ 睬育 $4 \mathrm{~h}$, 吸去液体, 加入 $150 \mu \mathrm{L}$ 的 DMSO, 振荡均匀, 酶标仪 $490 \mathrm{~nm}$ 处检测吸 光度值, 试验结果采用 SPSS16.0 统计软件计算 $\mathrm{IC}_{50}$ 值.

辅助材料(Supporting Information) 化合物 3, 5a $\sim 5 \mathbf{r}$ 的 ${ }^{1} \mathrm{H}$ NMR, ${ }^{13} \mathrm{C}$ NMR, HRMS 的谱图. 这些材料可以免 费从本刊网站(http://sioc-journal.cn/)上下载.

\section{References}

[1] Li, N.; Xin, J. C.; Ma, Q. S.; Li, E. D.; Meng, Y. Q.; Bao, C. N.; Yang, P.; Song, P. P.; Cui, F.; Cheng, P. J.; Gu, Y. F.; Zhao, P. R.; Ke, Y.; Liu, H. M.; Zhang, Q. R. Chin. J. Org. Chem. 2018, 38, 665 (in Chinese)

(栗娜, 辛景超, 马启胜, 李二冬, 孟娅琪, 包崇男, 杨鹏, 宋攀 攀，崔飞，陈鹏举，顾一飞，赵培荣，可钰，刘宏民，张秋荣，有 机化学, 2018, 38, 665.)

[2] Duan, Y. D.; Jiang, Y. Y.; Guo, F. X.; Chen, L. X.; Xu, L. L.; Zhang, W.; Liu, B. Fitoterapia 2019, 135, 114.

[3] Jayaraj, G.; Sherlin, H. J.; Ramani, P.; Don, K. R.; Santhanam, A.; Sukumaran, G.; Ramasubramanian, A. J. Oral Maxil. Surg. 2019, $31,228$.

[4] Ronald, C.; Chen, M. D.; M. P. H. Urol. Oncol. 2019, 5, 1.

[5] Zhang, X.; Zhu, Y.; Dong, S.; Zhang, A.; Lu, Y.; Li, Y.; Lv, S.; Zhang, J. Life Sci. 2019, 232, 1165.

[6] Wang, C. J.; Cao, Q. P.; Yang, H.; Song, P .P.; Xue, D. Q.; Cui, F.; Gu, Y. F.; Zhang, X. S.; Tian, Y. N.; Zhang, Q. R.; Liu, H. M. Chin. J. Org. Chem. 2016, 36, 1626 (in Chinese).

(王超杰, 曹钦坡, 杨慧, 宋攀攀, 薛登启, 崔飞, 顾一飞, 张孝 
松, 田亚楠，张秋荣，刘宏民，有机化学，2016, 36, 1626.)

[7] Li, J.; Pan, H.; Qiao, S.; Li, Y.; Wang, J.; Liu, W.; Pan, W. Int. J. Biol. Macromol. 2019, 134, 63.

[8] Kong, K.; Zhang, J.; Zhao, P.; Chen, J.; Chen, Y. Tetrahedron 2017, 73,6742 .

[9] Zhang, S.; Zhao, Y.; Liu, Y.; Chen, D.; Lan, W.; Zhao, Q.; Dong, C.; Xia, L.; Gong, P. Eur. J. Med. Chem. 2010, 45, 3504.

[10] Lu, X.; Peng, Y.; Wang, C.; Yang, J.; Bao, X.; Dong, Q.; Zhao, W.; Tan, W.; Dong, X. Eur. J. Med. Chem. 2017, 138, 384.

[11] Prakash, O.; Aneja, D. K.; Hussain, K.; Kumar, R.; Arora, S.; Sharma, C.; Aneja, K. R. J. Heterocycl. Chem. 2012, 49, 1091.

[12] Hashash, M. A. E.; Dalal, B. G.; Nayera, A. W.; Mohamed, A. K. J. Chem. Eng. Process Technol. 2014, $5,4$.

[13] Sun, X. Y.; Hu, C.; Deng, X. Q.; Wei, C. X.; Sun, Z. G.; Quan, Z. S. Eur. J. Med. Chem. 2010, 45, 4807.

[14] Zhang, H.; Zhang, H.; Tian, Y.; Quan, Z. Chin. J. Org. Chem. 2017, 37, 2322 (in Chinese) (张海明, 张洪健, 田玉顺, 金哲山, 有机化学, 2017, 37, 2322.)

[15] Xin, J. C.; Li, N.; Ma, Q .S.; Li, E.D.; Meng, Y. Q.; Ke, Y.; Liu, H. M.; Zhang, Q. R. Chin. J. Org. Chem. 2018, 38, 451 (in Chinese). (辛景超, 栗娜, 马启胜, 李二冬, 孟祥川, 可钰, 刘宏民, 张秋 荣, 有机化学, 2018, 32, 451.)

[16] Boraei, A. T. A.; Ashour, H. K.; El Tamany, E. S. H.; Abdelmoaty, N.; El-Falouji, A. I.; Gomaa, M. S. Bioorg. Chem. 2019, 85, 293.

[17] Mozafari, R.; Heidarizadeh, F. Polyhedron 2019, 162, 263.

[18] Ruiz-Schutz, V. C.; Gomes, L. M.; Mariano, R. C.; Almeida, D. V. P.; Maluf, F. C.; Schutz, F. A. Crit. Rev. Oncol. Hematol. 2019, 141, 163.

[19] To, K. K.; Poon, D. C.; Wei, Y.; Wang, F.; Lin, G.; Fu, L. W. Bio- chem. Pharmacol. 2015, 97, 27.

[20] Yang, H. M.; Park, C. W.; Park, S.; Kim, J. D. Colloids Surf., B 2018, 161, 183.

[21] Marochkin, I. I.; Dorofeeva, O. V. Comput. Theor. Chem. 2012, 991, 182.

[22] Rodriguez-Hernandez, D.; Barbosa, L. C. A.; Demuner, A. J.; Ataide Martins, J. P.; Fischer, L.; Csuk, R. Eur. J. Med. Chem. 2019, $168,436$.

[23] Oteyza, D. G.; Garcia-Lekue, A.; Vilas-Varela, M.; Merino-Diez, N.; Carbonell-Sanroma, E.; Corso, M.; Vasseur, G.; Rogero, C.; Guitian, E.; Pascual, J. I.; Ortega, J. E.; Wakayama, Y.; Pena, D. ACS Nano 2016, 10, 9000.

[24] Zhai, L.; Zhang, Y. L.; Kang, J. S.; Oelschlaeger, P.; Xiao, L.; Nie, S. S.; Yang, K. W. ACS Med. Chem. Lett. 2016, 7, 413.

[25] Modh, R. P.; Kumar, S. P.; Jasrai, Y. T.; Chikhalia, K. H. Arch. Pharm. (Weinheim) 2013, 11, 346.

[26] Ma, L.; Li, S.; Zheng, H.; Chen, J.; Lin, L.; Ye, X.; Chen, Z.; Xu, Q.; Chen, T.; Yang, J.; Qiu, N.; Wang, G.; Peng, A.; Ding, Y.; Wei, Y.; Chen, L. Eur. J. Med. Chem. 2011, 46, 2003.

[27] Li, Q.; Chen, P.; Yang, H.; Luo, M.; You, W.; Zhao, P. Chem. Pap. 2017, 72, 651 .

[28] Kumar, R.; Kaur, M.; Bahia, M. S.; Silakari, O. Eur. J. Med. Chem. 2014, 80,83 .

[29] Wang, G. B.; Wang, L. F.; Li, C. Z.; Sun, J.; Zhou, G. M.; Yang, D. C. Res. Chem. Intermed. 2011, $38,77$.

[30] Wang, G.; Peng, Z.; Wang, J.; Li, X.; Li, J. Eur. J. Med. Chem. 2017, 125, 423.

[31] Wang, J. H.; Luo, T. W.; Li, S. B.; Zhang, Y. B.; Wang, C. J.; Zhao, J. Curr. Med. Chem. 2013, 20, 4070. 